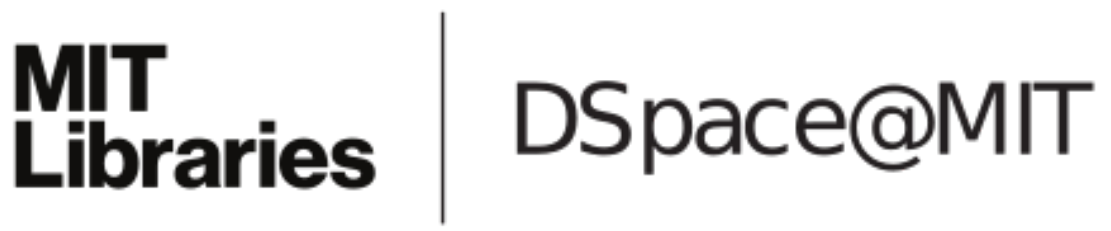

\author{
MIT Open Access Articles
}

\section{Continuous concentric lamellar block copolymer nanofibers with long range order}

The MIT Faculty has made this article openly available. Please share how this access benefits you. Your story matters.

Citation: Ma, Minglin et al. “Continuous Concentric Lamellar Block Copolymer Nanofibers with Long Range Order." Nano Letters 9.4 (2009): 1678-1683.

As Published: http://dx.doi.org/10.1021/n1900265y

Publisher: American Chemical Society

Persistent URL: http://hdl.handle.net/1721.1/68992

Version: Author's final manuscript: final author's manuscript post peer review, without publisher's formatting or copy editing

Terms of use: Creative Commons Attribution-Noncommercial-Share Alike 3.0 


\title{
Continuous Concentric Lamellar Block Copolymer Nanofibers with Long Range Order
}

Minglin Ma, ${ }^{1,3}$ Kirill Titievsky, ${ }^{1}$ Edwin L. Thomas, ${ }^{2,3}$ Gregory C. Rutledge ${ }^{1,3 *}$

${ }^{1}$ Department of Chemical Engineering, ${ }^{2}$ Department of Material Science and Engineering, ${ }^{3}$ Institute for Soldier Nanotechnologies, Massachusetts Institute of Technology, Cambridge, MA 02139, USA

* e-mail rutledge@mit.edu, tel: (617) 253-0171, fax: (617) 258-5766.

\begin{abstract}
Fibers with long-range ordered internal structures have applications in various areas such as photonic band gap fibers, optical waveguides, wearable power, sensors and sustained drug release. Up to now, such fibers have been formed by melt extrusion or drawing from a macroscopic preformed rod, and were typically limited to diameters $>10 \mu \mathrm{m}$, with internal features $>1 \mu \mathrm{m} .{ }^{1}$ We describe a new class of continuous fibers and fibrous membranes with long-range ordered concentric lamellar structure that have fiber diameters and feature sizes 2-3 orders of magnitude smaller than those made by conventional methods. These fibers are created through confined self-assembly of block copolymers within core-shell electrospun filaments. In contrast to the copolymer in bulk or thin films, the domains of the concentric lamellar structure are shown here to vary quantitatively with (radial) position, and to exhibit a novel dislocation that accommodates variations in fiber diameter robustly, permitting for the first time the realization of long range order in technologically meaningful, continuous fibers with approximately $300 \mathrm{~nm}$ diameter and $50 \mathrm{~nm}$ radial period.
\end{abstract}


The morphologies associated with the self-assembly of molecules have long been of interest in material science. ${ }^{2}$ Block copolymers are well-known examples of self-assembling, amphiphilic systems ${ }^{3}$ that are composed of chemically distinct and usually immiscible polymer blocks. From both fundamental ${ }^{4,5}$ and applied ${ }^{6,7}$ points of view, block copolymers have attracted interest due to their ability to form ordered morphologies with characteristic dimensions in the range of $10-100 \mathrm{~nm}$, dimensions that are hard to achieve by conventional, top-down technologies such as photolithography or extrusion. In bulk, $A / B$ diblock copolymers form periodic morphologies comprised of lamellae, bicontinuous cubic double gyroids, hexagonally packed cylinders or body-centered-cubic (bcc) packed spheres, depending on the copolymer molecular weight, the volumetric compositions of each polymer block and the interactions between respective monomers. When self-assembly is confined on a length scale comparable to the characteristic period of the copolymer domains, interesting new morphologies can be realized. In block copolymer thin films, the confinement effects and boundary conditions have been shown to result in either a higher degree of ordering of the phases, a change of the fundamental repeat period, or a shift of the phase boundaries between different morphologies. ${ }^{8}$ Additionally, external fields such as flow fields ${ }^{9}$ or electrical fields ${ }^{10}$ and lithographically defined templates ${ }^{11-13}$ can be used to direct the block copolymer self-assembly to achieve long range order.

Novel structures have been found to arise when block copolymers are confined in nonplanar geometries with diameter $(D)$ up to an order of magnitude larger than the bulk period $\left(L_{0}\right)$ of the copolymer. ${ }^{14-16}$ In particular, cylindrical confinement has been studied both theoretically ${ }^{17-23}$ and experimentally ${ }^{24-28}$ in this regard. For example, a concentric lamellar structure resembling the common myelin figure ${ }^{29}$ that arises from the unconstrained 
self-assembly of smectic liquid crystals in water was also observed recently by Russell et al. for a lamella-forming block copolymer that was confined in the cylindrical nanopores of an alumina membrane. ${ }^{24}$ The concentric lamellar morphology can be identified as a smectic A structure with a $\mathrm{s}=+1$ disclination defect line running along the cylinder axis. ${ }^{30}$ The same morphology was subsequently achieved in continuous fibers using a two-fluid, coaxial electrospinning technique, followed by thermal annealing of the fibers in which the block copolymer core self-assembles into the concentric lamellar morphology under the confinement of a rigid shell component. ${ }^{31,32}$ The continuous, filamentary nature of the electrospun, annealed fibers is novel and significant, from both science and engineering perspectives, as it offers the only form to date in which long range order along the axis of confinement is possible. By this process, continuous fibers can be produced at rates on the order of $0.1 \mathrm{~g}\left(10^{6}\right.$ meters $)$ of fiber per hour per jet, and the process is readily scalable to multiple jets. However, the persistence of flow-induced defects and lack of long range order in the early reports ${ }^{31,32}$ precluded a detailed understanding of the underlying physics of self-assembly under cylindrical confinement. Similarly, the porous alumina process produces only relatively short $(\sim 5 \mu \mathrm{m})$ nanorods, by which it is impossible to observe any phenomena with a length scale longer than the rod length, and has resulted so far in materials for which it has not been possible to quantify variations in domain sizes with radial position, or even whether such domains should be larger or smaller than those in the unconfined bulk lamellar morphology. ${ }^{24,28}$ This is in contradiction to simulations that predict domain sizes to vary with both pore size and radial position within the pore. ${ }^{19,21}$ The characterization of defects that perturb such morphologies is largely unexplored territory. Realization of the considerable potential for application of structured nanofibers in areas such as photonics, drug delivery and 
energy is contingent upon the availability of continuous fibers with uninterrupted, long range order over substantial distances and precise understanding and control of these domain sizes. $^{33}$

Here, we provide evidence for continuous block copolymer nanofibers with concentric lamellar structure and long range order, and resolve some of the critical issues mentioned above through the most quantitative analysis to date of domain sizes and defect formation along the fiber axis. We show that the lamellar structure confined in fibers differs significantly from that observed in the unconfined (i.e. bulk) lamellar morphology or that arising under confinement in thin planar films. ${ }^{34}$ In fibers, the morphology in the radial direction is not longer strictly periodic; instead, the domains vary significantly in size, with the outer domains being slightly smaller than the bulk value and the central domain being much larger. These observations are explained by the increase in interfacial energy arising from the increased curvature of the domains near the fiber axis, which leads in turn to a decrease in the interfacial chain density. A unique type of defect, a radial edge dislocation loop, is identified as the main mechanism by which the number of block copolymer domains changes to accommodate variations in the fiber diameter.

Fibers were obtained by co-axial electrospinning of a poly(styrene- $b$-dimethylsiloxane) (PS-PDMS) block copolymer as the core component and a poly(methacrylic acid) (PMAA) homopolymer as the shell, followed by annealing at $160^{\circ} \mathrm{C}$ for 10 days under vacuum. The PS-PDMS copolymer forms a lamellar morphology in bulk with a period $\left(L_{0}\right)$ of $56 \mathrm{~nm}$, as determined by small angle X-ray scattering (SAXS).

A mat composed of the PS-PDMS/PMAA core/shell electrospun fibers is shown in Fig. 
1a, b. Long continuous fibers of PS-PDMS (Fig. 1c) can be easily produced by removal of the PMAA shell using methanol as the selective solvent. The average diameter of the as-spun core/shell fibers is $800 \pm 150 \mathrm{~nm}$, while that of the PS-PDMS core fibers is $300 \pm 220 \mathrm{~nm}(\sim$ 1-10 $\left.L_{0}\right)$ after removal of the shell. The variations of fiber diameter occur over runs of fiber on the order of 1-10 cm in length, roughly five orders of magnitude greater than the diameters themselves, and are thus extremely gradual in the axial direction; as discussed later, this is consistent with the sparseness of dislocation defects along the length of the fiber. A well-defined concentric lamellar structure is formed within the fiber core after annealing, as shown by Fig. 1d-f. Fig. 1e also shows that the PS block preferentially segregates to the core/shell interface with PMAA due to its lower Flory interaction parameter $\left(\chi_{\mathrm{PS} / \mathrm{PMAA}}=0.14\right.$ at $\left.160{ }^{\circ} \mathrm{C}\right)$ compared to that of PDMS with PMAA ( $\chi_{\mathrm{PDMS} / \mathrm{PMAA}}=0.72$ at $\left.160{ }^{\circ} \mathrm{C}\right) .{ }^{35}$ As expected, this outermost PS is a monolayer and is approximately half as thick as the inner PS domains, which are bilayers. Some control over the morphology of the fibers can be exerted by tailoring the interactions between the shell material and the polymer blocks. (See Fig. S1 in Supporting Information for one example.)

To understand quantitatively the effect of confinement on the concentric lamellar structure, we first show how the total number of bilayers $(N)$ varies as a function of the diameter $(D)$ of the PS-PDMS core, as illustrated in Fig. 2. $D / N$ is the average thickness of a complete bilayer of PS and PDMS. The degree of confinement can be expressed in terms of the ratio, $D / L_{0}$; a smaller $D / L_{0}$ corresponds to a greater degree of confinement. As $D$ increases, $N$ increases in a discrete manner. However, $N$ is not determined uniquely by $D / L_{0}$; different numbers of bilayers may be observed at the same degree of confinement (e.g $N=6$ or 7 for 
$D / L_{0} \sim 6.5$ ). Similarly, for a given $N$, a range of PS-PDMS core diameters $(D)$ may be observed. For fibers with $D / L_{0}>5$, the values of $D / N$ are distributed more or less statistically about the bulk value $L_{0}$, which may explain why previous works ${ }^{19,24,28}$ have reported domains that may be either compressed or expanded relative to bulk. However, for the smaller diameter fibers, where confinement is greatest $\left(D / L_{0}<5\right)$, the data fall predominantly below the line, indicating that these domains are significantly expanded relative to bulk. This trend points to a systematic deviation of the domain size from the average. In this respect, the concentric lamellar morphology differs qualitatively from that observed in thin films.

To explore this systematic deviation more closely, we show in Fig. 3a the variation of domain size as a function of domain number, counting from the axis of the fiber outward. A domain is defined here as a cylindrical region of pure PS or PDMS between two successive inter-material dividing surfaces (IMDS). The domain sizes in Fig. 3a are normalized by their unconfined bulk values for PS ( $34 \pm 2 \mathrm{~nm}$, by TEM) and PDMS ( $22 \pm 2 \mathrm{~nm})$, respectively. This figure shows unambiguously that the sizes of outer domains $\left(d_{n}, n>1\right)$ are slightly smaller than the bulk value, and that they approach the bulk value with increasing domain number. Meanwhile, the central domain $\left(d_{1}\right)$ ranges from 8 to $76 \%$ larger than the bulk value. The unique behavior of the central domain explains why the $D / N$ is systematically larger than $L_{0}$ for the smallest fibers. The trend of domain sizes observed here is consistent with previous simulation results using a simulated annealing technique ${ }^{21}$ and further confirmed by simulations based on a coarse grained bead-spring model. (See Fig. S2.)

The reason for the difference in size of the central and outer domains can be understood 
as follows. We treat the PS-PDMS block copolymer chain very simply as comprising two strongly segregated brushes, $A$ and $B$, of equal length, joined together at the $A B$ interface (IMDS; see Fig. 3b) so that the bulk domain size for each block is $L_{0} / 2$. In bulk, equating the number of copolymer blocks within a domain bounded by two successive $A B$ interfaces with the number of copolymer chains crossing each of the two interfaces (of equal area) leads to the following equation,

$$
s_{0}=\rho_{0} L_{0} / 2
$$

where $L_{0}, s_{0}$ and $\rho_{0}$ are the bulk period (i.e. the thickness of an $A B$ bilayer), interfacial chain density (number of chains per unit interfacial area) and packing density (number of chains per unit volume), respectively. Applying a similar conservation of the number of polymer blocks per unit length of fiber to the central domain and outer cylindrical shells depicted in Fig. 3b, we obtain

$$
s_{1}=\rho_{0} r_{1}
$$

for the central domain and

$$
s_{i} r_{i}+s_{i+1} r_{i+1}=\rho_{0}\left(r_{i+1}^{2}-r_{i}^{2}\right), i=1,2,3 \ldots
$$

for the outer domains. $s_{i}$ is the interfacial chain density at the $i^{\text {th }}$ interface counting from the center of the fiber (c.f. Fig. 3b). In the two equations above, the polymer is assumed to have the same packing density in the fibers as in bulk; the compressibilities of PS and PDMS are approximately $2 \times 10^{-10} \mathrm{~Pa}^{-1}$ and $6 \times 10^{-10} \mathrm{~Pa}^{-1}$, respectively, rendering them essentially incompressible under these conditions. ${ }^{37}$

Equations (1) to (3) show that the domain sizes are related to the interfacial chain densities. We consider two limiting cases for an incompressible system: bulk copolymer-like interfacial chain density and bulk copolymer-like domain spacing. Taking first the case of 
bulk-like interfacial chain density, i.e. $s_{i}=s_{0}$, we obtain $r_{1}=0.5 L_{0}$ and $r_{i+1}-r_{i}=0.5 L_{0}$ or equivalently, $d_{1}=2$ and $d_{n>1}=1$. This result indicates that the domain in the center must be significantly expanded to maintain bulk-like block density and bulk-like interfacial chain density. Intuitively, it is a result of the excluded volume interactions among the polymer blocks in the central domain that would otherwise be "crowded" by the singularity in volume at the axis of the fiber. However, expansion of the domain requires additional stretching of the polymer blocks confined in the center, over and above that arising from localization of the diblock junction at the IMDS, and is resisted by chain conformational entropy. In addition, there is a reduction of conformational entropy associated with the curvature of the IMDS, which further restricts the range of angles the chain is allowed to explore on the concave side of the IMDS. (See Fig. S3 for further details.)

On the other hand, taking the case of domain spacing comparable to that in the bulk copolymer morphology, (i.e. $d_{1}=d_{n>1}=1$ or $r_{1}=0.25 L_{0}$ and $\left.\left(r_{i+1}-r_{i}\right)=0.5 L_{0}\right)$, the interfacial chain density at the first IMDS must decrease by a commensurate amount (i.e. $s_{1}=$ $\left.0.5 s_{0}\right)$ in order to maintain bulk-like block density within the domain. This results in an increased interfacial energy per chain. The curvature induced conformational entropy loss associated with the central domain is also larger in this case, compared to the previous case where the interfacial chain density is assumed to be equivalent to that in the bulk copolymer, due to the increased curvature required of the first IMDS. The set of experimental values observed for the size of the central domain (Fig. 3a) lies between the bulk value, $d_{l}=1$, and $d_{l}=2$ obtained for the case of bulk copolymer interfacial chain density, and can be explained simply as a consequence of the variation of the overall fiber diameter and the trade-off between the reduced conformational entropy due to space-filling and curvature, and increased 
interfacial energy due to depletion of block junctions. This is similar to the case of bulk block copolymer, where the balance between the chain entropy and interfacial energy has been used to estimate the lamellar period in the strong segregation regime. ${ }^{38}$ This interpretation is also consistent with the classical theory of curvature elasticity of block copolymer monolayers, in which increased curvature leads to an increase in the interfacial area per chain, or decrease in the interfacial chain density, compared to the bulk copolymer. ${ }^{39,40}$

For the outer domains, the model suggests that the domain sizes assume the bulk value if $s_{i}=s_{0}$, implying that the lower curvatures do not affect the sizes of the outer domains. Of course, bending the intrinsically flat interface of the lamellar morphology is energetically unfavorable, ${ }^{39,40}$ resulting in a slightly decreased interfacial chain density, as demonstrated most dramatically for the central domain discussed above. This reduction in interfacial chain density consequently permits a decrease of the domain sizes and a recovery of conformational entropy, as the crowding of the brush-like interface lessens and the chains more closely approach their preferred random coil conformations; this is consistent with the experimental data, in which the outer domains are slightly smaller than bulk value. As the domain index increases, the bending becomes less significant and the domains approach their bulk characteristics.

With respect to formation of fibers with long range radial and axial order, the important question is the mechanism by which the concentric lamellar morphology is interrupted and defects are formed as the number of domains in the radial direction varies along the length of the fiber. The unique behavior of the central domain offers some insight into this question. Taking advantage of the long continuous nature of electrospun fibers, we can locate and examine transitions in the nature of the domain morphology as the diameter of the PS-PDMS 
core fiber varies. Fig. 4a, b show two representative longitudinal views of the concentric lamellar structure near these transitions. Based on frequency of observation over a large number of TEM images, such transitions almost always involve the conversion of the central domain from $A$ to $B$ or $B$ to $A$ on the axis of the fiber.

Based on this, several important observations can be made. First, for a given number of domains, as the diameter $D$ of the core fiber undulates very gradually along the length of a fiber (e.g. as indicated by the arrow in Fig. 4a), the small variations in diameter are absorbed almost entirely by the central domain, while the thicknesses of the outer domains stay approximately the same. This is evident in the plot in Fig. 3a, where the central domain is shown to have a much larger variation in thickness than the outer ones. Second, when the diameter of the core fiber increases sufficiently, an additional domain inserts within the overly-expanded central domain to relax the unusually large stress experienced by that domain. This phenomenon is very similar to the formation of an edge dislocation in smectic A liquid crystals. ${ }^{41}$ Taken in cross section (Fig. 4c), the edge dislocation can be identified by the Burgers vector (b) oriented radially and orthogonal to the dislocation core tangent line vector (t); the dislocation core itself is curved, and describes a circumferential loop that closes upon itself. We call this a "radial edge dislocation loop". The fact that the direction of the Burgers vector of the dislocation varies is a consequence of the presence of the $\mathrm{s}=+1$ disclination line defect along the fiber axis. In the limit that the dislocation core is confined to the central domain, as shown in Fig. 4d, the loop itself is singular. This type of defect is expected to be energetically more favorable than the one in Fig. $4 \mathrm{c}$ because the dislocation loop is shorter in length and the associated excess strain energy should be less. Finally, and most importantly, the defect tends to be localized around the central domain- that is, all 
domains except the central one remain continuous without interruption over macroscopic length scales. Indeed, $1 \mu \mathrm{m}$ long sections of defect-free fiber, where even the central domain is uninterrupted, are readily observed by TEM (Fig. 4e, f), indicating that such defects are relatively rare. (See Fig. S4 for more images. Also see Fig. S5 for data on a second PS-PDMS block copolymer.) Based on frequency of observation and the slow modulation of fiber diameter, we estimate an average defect spacing along the fiber axis of about 1-3 $\mu \mathrm{m}$ in our fibers. This spacing can be modified through control of the block copolymer fiber core diameter during fabrication.

In summary, long continuous fibers having concentric lamellar morphology and long range order have been achieved by the fabrication of core-shell nanofibers, using two-fluid coaxial electrospinning, followed by confined self-assembly of a PS-PDMS block copolymer within the core. The cylindrical confining geometry is shown to alter the domain sizes of lamella-forming block copolymers in a way that is remarkably different from confined thin films, where the period is constant across the film thickness. In the cylindrical geometry, the central domain is always much ( $\sim 40 \%$ in average) larger than the bulk value, yet smaller than the value estimated by assuming interfacial chain density equivalent to bulk; the outer domains are always slightly $(<10 \%)$ smaller than the bulk value. The thicknesses of both the central and outer domains can be explained by a reduction in interfacial chain density imposed by the curvature of the IMDS associated with the cylindrical geometry. The study also shows that radial edge dislocation loops may form to accommodate variations in the core fiber size, with the outer domains remaining continuous and ordered over long lengths of fiber; this long range order can be improved through tight control of fiber core size (e.g. by 
adjusting the solution properties and optimizing the operating parameters in electrospinning).

The availability of this new class of continuous nanofibers having coherent, long ranged order, and the results reported here, create numerous opportunities for further studies of both fundamental and practical nature. For example, there exists considerable freedom to control both the structural properties (e.g. domain sizes), by adjusting the molecular weight of the copolymer (Fig. S5), and the chemical nature of the material by simply choosing different core diblock or shell homopolymer compositions (Fig. S1). These can in principle be used to modulate the stability and frequency of radial edge dislocation loops within the fibers. Understanding and control of these aspects of self-assembly under cylindrical confinement could lead to a tremendous expansion above and beyond the current list of applications for continuous nanofibers ${ }^{42,43}$. For example, long-range ordered fibers may be used for sustained and controllable drug release by loading multiple drugs selectively into different domains of the fibers. These fibers may also serve as low-loss optical waveguides by choosing domain components with high refractive index contrast.

\section{Acknowledgements}

The work was supported in part by the U.S. Army through the Institute for Soldier Nanotechnologies (ISN) at MIT, under Contract DAAD-19-02-D-0002 with the U.S. Army Research Office. We thank Dr. A. Avgeropoulos and Dr. R. M. Hill for providing us the PS-PDMS block copolymer, and Dr. M. Kleman for helpful discussions concerning defects in smectics.

\section{References}

1. Abouraddy A. F., et al. Nature Mater. 2007, 6, 336.

2. Whitesides, G. M.; Grzybowski, B. Science 2002, 295, 2418.

3. Bucknall, D. G.; Anderson, H. L. Science 2003, 302, 1904.

4. Hamley, I. W. The physics of block copolymers (Oxford University Press, Oxford, 1998).

5. Fredrickson, G. H.; Bates, F. S. Annu. Rev. Mater. Sci. 1996, $26,501$.

6. Park, C., Yoon, J., Thomas, E. L. Polymer 2003, 44, 6725.

7. Forster, S.; Plantenberg, T. Angew. Chem. Int. Ed. 2002, 41, 688.

8. Fasolka, M. J.; Mayes, A. M. Annu. Rev. Mater. Res. 2001, 31, 323. 
9. Chen, Z.; Kornfield, J. A.; Smith, S. D.; Grothaus, J. T.; Satkowski, M. M. Science 1997, $277,1248$.

10. Thurn-Albrecht T. et al. Science 2000, 290, 2126.

11. Kim S. O. et al. Nature 2003, 424, 411.

12. Cheng, J. Y.; Ross, C. A.; Smith, H. I.; Thomas, E. L. Adv. Mater. 2006, 18, 2505.

13. Bita I. et al. Science 2008, 321, 939.

14. Thomas, E. L.; Reffner, J. R.; Bellare, J. Colleque De Physique, Colleque 1990, C7-363.

15. Yabu, H.; Higuchi, T.; Shimomura, M. Adv. Mater. 2005, 17, 2062.

16. Arsenault A. C. et al. J. Am. Chem. Soc. 2005, 127, 9954.

17. He, X.; Song, M.; Liang, H.; Pan, C. J. Chem. Phys. 2001, 114, 10510.

18. Sevink, G. J. A.; Zvelindovsky, A. V.; Fraaije, J. G. E. M.; Huinink, H. P. J. Chem. Phys. 2001, 115, 8226.

19. Li, W.; Wickham, R. A.; Garbary, R. A. Macromolecules 2006, 39, 806.

20. Yu B. et al., Phys. Rev. Lett. 2006, 96, 138306.

21. Yu B. et al. J. Chem. Phys. 2007, 127, 114906.

22. Wang, Q. J. Chem. Phys. 2007, 126, 24903.

23. Sevink, G. J. A.; Zvelindovsky, A. V. J. Chem. Phys. 2008, 128, 084901.

24. Xiang H. et al. Macromolecules 2004, 37, 5660.

25. Shin K. et al. Science 2004, 306, 76.

26. Wu Y. et al. Nat. Mater. 2004, 3, 816.

27. Xiang H. et al. J. Polym. Sci: Part B Polym. Phys. 2005, 43, 3377.

28. Sun Y. et al. Macromol. Rapid Commun. 2005, 26, 369.

29. Sakurai, I.; Suzuki, T.; Sakurai, S. Biochim. Biophys. Acta 1989, 985, 101.

30. Kleman, K. Points, Lines and Walls : In Lquid Crystals, Magnetic Systems and Various Ordered Media. (Wiley, Chichester-New York-Toronto-Singapore, 1983), pp. 322.

31. Kalra V. et al. Adv. Mater. 2006, 18, 3299.

32. Ma, M.; Krikorian, V.; Yu, J. H.; Thomas, E. L.; Rutledge, G. C. Nano Lett. 2006, 6, 2969.

33. Hart S. D. et al. Science 2002, 296, 510.

34. Turner, M. S. Phys. Rev. Lett. 1992, 69, 1788.

35. 'Polymer Handbook' (Eds J. Brandrup, E. H. Immergut and E. A. Grulke). 4th Edn, Wiley, New York, 1999, P. VII/675.

36. Bates, F. S.; Berney, C. V.; Cohen, R. E.; Wignall, G. D. Polymer 1983, 24, 519.

37. Fakhreddine, Y. A.; Zoller, P. J. Appl. Polym. Sci. 1990, 41, 1087.

38. Helfand, E.; Wasserman, Z. R. Macromolecules 1976, 9, 879.

39. Wang, Z.; Safran, S. A. J. Chem. Phys. 1991, 94, 679.

40. Gido, S. P.; Thomas, E. L. Macromolecules 1994, $27,849$.

41. Williams, C. E.; Kleman, M. J. De Physique, Colleque 1975, C1-315.

42. Greiner A.; Wendorff, J. H. Angew. Chem. Int. Ed. 2007, 46, 5670.

43. Yu, J. H.; Rutledge, G. C. Electrospinning. in Encycl. Polym. Sci. Tech. 2007.

\section{Supporting Information available:}

Details of methods; Supplemental Figures 1-5. 
Fig. 1. A multi-scale view of an electrospun block copolymer fiber mat. a, A macroscopic image of the PS-PDMS/PMAA fiber mat (scale bar $=1 \mathrm{~cm}$ ). b, Scanning electron microscopy (SEM) image of the as-spun core/shell fibers (scale bar $=10 \mu \mathrm{m}$ ). c, SEM image of the PS-PDMS core fibers after removal of the PMAA shell using methanol (same magnification as b). d, e, Cross sectional transmission electron microscopy (TEM) images of the fibers after annealing, showing the core/shell structure and concentric lamellar structure in the core; in e, the dark layers are identified to be PDMS due to its higher electron density, and the light layers are PS. The region surrounding the PS-PDMS core is the PMAA shell. f, A tilt TEM image of a PS-PDMS core, showing a 2D projection of the 3D concentric lamellar structure. Note that the outermost PS monolayer is not resolved in this image due to the lack of sufficient contrast between PS and PMAA in this case.

Fig. 2. Total number $(N)$ of block copolymer bilayers as a function of degree of confinement $\left(D / L_{0}\right)$. The red line is a reference line based on the morphology of the unconfined bulk: $N=D / L_{0}$. The blue circles are data points from different TEM cross sections of electrospun fibers. $D$ is defined as the diameter of the PS-PDMS component of the core/shell fibers. A representative TEM image is inserted to illustrate the structure for several specific $N$. (All the images are presented at the same magnification.) Cross sections with an odd number of bilayers have PDMS as the central domain, while those with an even number of bilayers have PS as the central domain.

Fig. 3. Domain sizes of concentric lamellar structure. a, Dependence of domain thickness $d_{n}$ on domain index, $n$, where $d_{n}$ is defined as the (nondimensional) distance between successive $A B$ interfaces ( $A=\mathrm{PS}$ and $B=\mathrm{PDMS}$ ), counting from the central domain outward, relative to that in the bulk. The outermost PS domain is a monolayer and is approximately half as thick as the other PS domains, so it is not included in the plot. b, From left to right, schematics for block copolymer chains in bulk and in a fiber (axial view). (Note: the chain configurations drawn here are for illustrative purposes only and are not intended to represent actual or average configurations. ${ }^{36}$ )

Fig. 4. Dislocation and long range order in concentric lamellar structure. a, b, Longitudinal views of the concentric lamellar structure near a fiber diameter transition where the number of bilayers increases by one. (Scale bar $=100 \mathrm{~nm}$ for $\mathbf{a}$ and $\mathbf{b}$ ) $\mathbf{c}$, d, Schematic illustrations for the radial edge dislocation with dislocation core line of nonzero and zero (effective) length, respectively. The arrow lines in c show a Burgers circuit with the Burgers vector $(\boldsymbol{b})$ from the start $(\mathrm{S})$ to the finish $(\mathrm{F})$. $\boldsymbol{b}$ is everywhere normal to the tangent vector of the loop $(t)$ depicting a radial edge dislocation. In $\mathbf{c}$, two bilayers are inserted and the domains are therefore more compressed after the insertion, compared with the dislocation structure in $\mathbf{d}$, where only one bilayer is inserted, for fibers of equal diameter. $\mathbf{e}, \mathbf{f}$, Longitudinal views of sections of the concentric lamellar structure with no interruption. (Scale bar $=100 \mathrm{~nm}$ for $\mathbf{e}$ and $\mathbf{f}$ ) 